\title{
Ethical considerations in neurology during the COVID-19 pandemic
}

\author{
Mohammadreza Tabary $^{1} \cdot$ Ruhollah Abolhasani $^{2} \cdot$ Farnaz Araghi $^{3} \cdot$ Armin Aryannejad $^{1} \cdot$ Behnam Safarpour Lima $^{4,5}$ (D)
}

Received: 20 October 2020 / Accepted: 27 December 2020 / Published online: 2 January 2021

(C) Fondazione Società Italiana di Neurologia 2021

\begin{abstract}
Coronavirus disease 2019 (COVID-19) pandemic has struck many countries and caused a great number of infected cases and death. Healthcare system across all countries is dealing with the increasing medical, social, and legal issues caused by the COVID-19 pandemic, and the standards of care are being altered. Admittedly, neurology units have been influenced greatly since the first days, as aggressive policies adopted by many hospitals caused eventual shut down of numerous neurologic wards. Considering these drastic alterations, traditional ethical principles have to be integrated with state-of-the-art ethical considerations. This review will consider different ethical aspects of care in neurologic patients during COVID-19 and how this challenging situation has affected standards of care in these patients.
\end{abstract}

Keywords COVID-19 $\cdot$ Neurology $\cdot$ Ethics $\cdot$ Ethical consideration

\section{Introduction}

Coronavirus disease 2019 (COVID-19) pandemic has struck more than 200 countries and caused a great number of infected cases and death so far. Healthcare system across all countries is dealing with the increasing medical, social, and legal issues exerted by the COVID-19 pandemic, and the standards of care are being refined to remedy the situation [1]. This situation has led to uncertainty into patient care, and the so-called rudimentary dictum of "just do the best you can" is considered to be suboptimal for systematically handling the situation [2]. Although a general ethical framework to a pandemic has been addressed by Hastings Center's as a 3-tiered approach (the

Behnam Safarpour Lima

drbehnam_safarpour@yahoo.com; b.safarpour@sbmu.ac.ir

1 Experimental Medicine Research Center, Tehran University of Medical Sciences, Tehran, Iran

2 School of Medicine, Tehran University of Medical Sciences, Tehran, Iran

3 Skin Research Center, Shahid Beheshti University of Medical Sciences, Tehran, Iran

4 Department of Neurology, Shahid Beheshti University of Medical Sciences, Tehran, Iran

5 Imam Hossein Medical and Educational Center, Madani St., Tehran, Iran duty to plan, the duty to safeguard, and the duty to guide) [3], meticulous guidelines for each specialization field will help physicians to uplift their practice level in concordance with ethical standards.

Admittedly, neurology units have been influenced greatly since the initial days of this pandemic, as aggressive policies adopted by many hospitals compelled the relocation of many beds and eventual shut down of numerous neurologic wards [4]. Considering these drastic alterations, traditional ethical principles seem not to be functional in some cases, and state-of-the-art ethical considerations should be introduced. In addition, patients with neurodegenerative and neuropsychiatric disorders and patients with neurologic disabilities are highly vulnerable in this situation. These patients might be dependent on others for their personal needs. Also, some neurologic diseases, such as dementia, are more common in advanced age groups, and these clusters live in residential homes with higher risk of COVID-19 transmission [5]. On top of the magnified social risk of stigma imposed on these patients [6], many of them have been excluded from hospitals to guarantee the adherence to resource allocation guidelines developed initially by many institutions [7]. Consequently, many of this fragile group of patients may face unjustified discrimination in healthcare decisions of scarcity [8] in the near future. Herein, we will consider different aspects of ethics regarding patients with neurological disorders, but first we start with a comparison between the contemporary and prior conditions. 


\section{What has changed and what has remained static?}

The principles of ethics should be maintained even during critical times. These standards include respect for persons, respecting patients' wishes especially for end-of-life medical decision making, and not imposing undesired invasive treatment on patients [5]. Furthermore, precluding patients solely based on our subjective assessment of their quality of life or their cognitive status are among pitfalls which can be brought about by scarce resource allocation policy, and it is considered as unjustified discrimination [5].

On the other hand, shortage of resources and facilities may change some ethical principles or the way they are interpreted. Short-term survival is being prioritized by all ethical guidelines [9-11], while long-term survival considerations seem to be diverse [9-11]. Although a shared intuition is that young patients should predominate over the elderly, this is disputable among stakeholders [11, 12].

What is widely acknowledged is that each ethical framework must engage and reflect the views of public and at the same time address the priorities of the society [5]. But a word of caution, patients with cognitive disorders, such as dementia, are more susceptible to be excluded from receiving proper care since they are less able to advocate for themselves compared to subjects with other comorbid conditions such as malignancies [5].

\section{What ethical considerations should still be included in all frameworks?}

\section{Flattening the curve}

As we are amid a pandemic, concepts of public health ethics ought to be addressed [13]. To comply with this, non-essential in-person visits and data collection for research projects can be postponed, and telemonitoring of patients may be a proper alternative [14].

\section{Emotional support}

Psychosocial support is becoming an important component of neuropalliative service in many neurological diseases, such as stroke and chronic neurodegenerative diseases [15]. Considering the profound psychosocial impact of this pandemic on patients [14], emotional well-being of our patients should be maintained regardless of being affected by COVID19. In addition, patients involved in clinical research are preoccupied by their dual roles as both patients and subjects [16]. Thus, emotional support should be provided even through online applications or phone [14]. On top of these issues, hospitals have banned visitors except under certain circumstances. No doubt will this restriction result in painful experiences [17] for both COVID-19 patients and others including patients admitted with neurological disorders. Many patients have died alone [18], and many non-COVID cases are unable to contact their families [19]. To resolve this problem, regular contact between patients and family member using online applications may mitigate this emotional burden. However, as mentioned in a previous study, $56 \%$ of attendees in a tele-neuropsychology webinar were not comfortable with the ethics of providing video technology for cognitive testing [20]. Thus, further studies are needed to ensure ethical considerations for tele-neurology.

\section{Non-abandonment}

There are two valid arguments on this issue. On the one hand, neuropalliative care is a new and evolving field within neurology, which is crucial to patients with severe neurologic illnesses [21]. Dumping these patients in the middle of this pandemic may breed adverse outcomes. On the other hand, many patients are involved in clinical trials and neurology researchers should endeavor to maintain necessary contact with the subjects.

\section{Scarcity caused by the pandemic: is it a battle for ventilators?}

Roughly $20 \%$ of all patients needing mechanical ventilation suffer from neurological dysfunction [22]. As limited number of critical care resources are present, ventilators have become one of the most crucial issues of the pandemic. To date, some ethical frameworks have been released. A team from Johns Hopkins University generated a guideline on two major ethical principles: short-term survival with the support of available resources and long-term survival with consideration of comorbidities [23]. Also, six ethical principles have been introduced: maximizing advantages including using limited resources responsibly and saving more lives or years of life, prioritizing COVID-19 resources to healthcare workers, ensuring equality using random allocation or lottery, thoughtful consideration of resource allocation, prioritizing those who have took part in COVID-19 experimentations, and providing equal resources to those with COVID-19 and those with other medical conditions [24]. However, maximizing the number of saved lives does not accompany a defined level of quality of life, as it can be difficult to assess and sometimes slanted [24]. It should also be mentioned that infection with COVID-19 should not guarantee priority by itself [25], as a high rate of mortality has been reported for those COVID-19 cases requiring mechanical ventilation [26].

Scarce resource allocation protocol (triage protocols) is considered to be helpful during crises [27]. These protocols 
may obligate removal of ventilator from one patient to benefit others, but at the same time they may accompany moral distress [28]. Mortality should be considered in these protocols to maximize the use of resources. It has been suggested to use prognostic instruments for neurologic diseases to assess the priority of resource allocation in these patients (such as the CPC scale for hypoxic-ischemic brain injury, the Glasgow Outcome Scale, the Hunt-Hess Scale or World Federal of Neurosurgery Aneurysm Severity Score for aneurysmal subarachnoid hemorrhage, the IMPACT score for traumatic brain injury, the NIH Stroke Scale for ischemic stroke, and the ICH Score for hemorrhagic stroke) [27]. However, considering mortality to allocate resources in neurology patients may give rise to disability discrimination. Neurological patients, such as patients with advanced dementia, minimally conscious state, and persistent vegetative state, may survive for years with limited consciousness and low mortality rates, and this approach will prioritize them over others [27]. To tackle this issue, using physiologically based variable protocols is encouraged [29]. What is widely agreed is that final decision should not be made on race, gender, ethnicity, socioeconomics, or perceived social worth $[23,30]$. To achieve this goal, independent triage teams are required including intensivists, clinical ethicists, nursing representatives, health system administrator, and legal counsel [27, 29].

\section{How has this pandemic affected neurologic patients?}

\section{Chronic neurologic disease}

Neurologists are now supposed to discuss with chronic patients the potential repercussion of interacting with a healthcare system facing many difficulties these days [27]. They ought to discuss the risk of being infected by COVID19 and the possibility of postponing unnecessary procedures [27]. However, both the burden and future sequelae of these chronic diseases should be taken into account [4]. On top of that, these patients may need admission due to specific medical emergencies, which should be considered by dedicating adequate number of beds in hospitals [4].

Current pandemic resulted in an unconscious superiority of young patients to older ones. As many neurological diseases are associated with ageing (such as dementia), these patients may receive less care amid this pandemic due to resource allocation policies [24, 31]. But it is worth paying attention to this saying: "When this pandemic ends and humanity survives, how will older adults view the rest of us?" [32]. In this regard, some principles have been introduced for patients with dementia. Decisions regarding these patients should be individualized, and they should be able to indicate their wishes about goals of care. Also, decision regarding resource allocation should respect their personhood, and these decisions should be based on expected survival rather than assumption about individual quality of life. In the meanwhile, prognostication should be assessed objectively using mortality risk, frailty, or functional disease stage. Finally, mild cognitive impairment should not be used to allocate lifesaving care, and palliative care should also be provided [33]. Telemonitoring can also be utilized in patients with cognitive impairment (such as Alzheimer's disease), whereas informed consent, informant history, and attention to privacy and autonomy should be taken into account [34]. Sleep disorders can also be monitored using tele-neurology, but ethical issues such as confidentiality and explicit consent should be addressed, and patients should not be forced to adhere to such programs [35].

Patients with multiple sclerosis (MS) are believed to be at a higher risk of infections due to the consumption of immunosuppressive drugs [36]; however, this higher vulnerability to such drugs during COVID-19 has been debatable [37]. MS patients seem to be completely aware of the current pandemic adhering to home quarantine [38]. The progressive form of the disease appears to accompany more disability rates, and thus, it needs more healthcare, visiting professionals, or referring to a hospital. This goal can also be achieved using Telemonitoring [39].

\section{Stroke}

Stroke admissions seem to have fallen all over the world during the current pandemic [40, 41]. Although some changes in stroke management are inevitable, the usual care should be provided [42]. Patients who are either suspected or confirmed cases of COVID-19 should not be disqualified from receiving individualized stroke care. Also, goals of care should be discussed for patients at risk of severe outcome [43]. In case of crisis, the crisis status should be recorded in electronic medical recordings, and EMS services should be notified. Vascular neurology and/or neurocritical care representation ought to be present in triage committees so that they can provide accurate long-term prognostic information for stroke cases [42].

\section{Neuromuscular disease}

Respiratory complications of the COVID-19 have raised concerns about the consequences on patients with chronic neuromuscular diseases, especially children and young adults [44]. Early in the beginning of this pandemic, pediatric neuromuscular disorders were categorized as low priority for critical resource allocation [9]. Many guidelines have been introduced as triage protocols, but they, for the most part, take into account short-term survival and are designed for specific populations. Thus, they fail to account for diversity which is 
Principles of Ethics

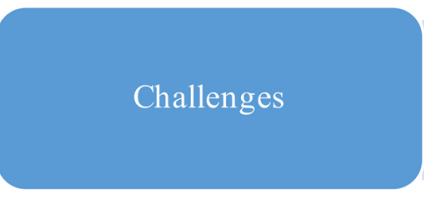

- Short-term vs. long-term surviva

- Young vs. elderly patients

- Poor prognostic illnesses and chronic neurologic diseases

- Scarcity casued by the pandemic

- Healthcare workers' safety and medical education during the pandemic

\section{What can be done?}

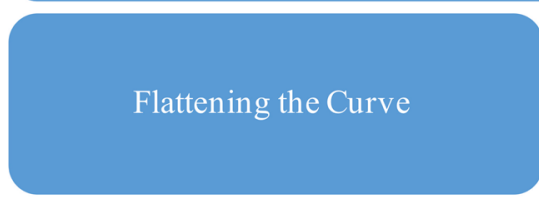

Emotionional Support

Non-abandonment

Scarcity caused by the pandemic: A battle for ventilators

\section{Chronic Neurologic Disease}

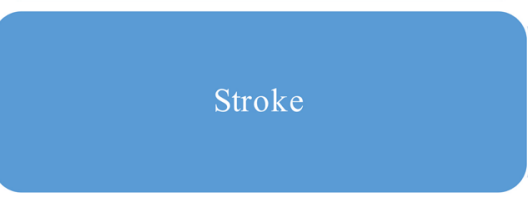

Neuromascular Disease
- Postponing non-essential in-person visits and data collection for research projects

- Implementing telemonitoring tools

- Consideing psychological impacts of the pandemic on patients

- Supporting patients and subjects of research projects emotionally through phone calls, online applications, and implementing tele-visiting

- Neuropalliative care for severe illnesses

- Maintain contact with subjects of research projects

- COVID-19 infection should not prioritize patients by itself.

- Prognostic neurologic instruments would be helpful in prioritizing neurologic patients, using physiologically-based-variables protocols.

- Race, gender, ethnicity, socioeconomics, or preceived social worth should not be

determinants in resource allocation.

- Discussing the possibility of postponing unnecessary procedures with patients - Considering enough beds for possible emergent cases

- In case of resource allocation, decision should be made objectively using morality risk, frailty, or functional disease stage.

- Usual care should be provided regardless of COVID-19 infection.

- Crisis status sould be recorded in electronic medical recordings.

- Neurocritical care representations in triage committees should be provided.

- Existing or future disabilities should not be a determinant in resource allocation

- Using transparent and accessible triage algorithms, especially in children, would be helpful.

- They might require ICU admission, and they should receive equal care with COVID-19 patients in ICUs.

- Decision should be made individually, and palliative consultation is necessary.

- Should be weighted against other uses of scarce medical resources.

- Should be avoided in unstable, critically ill patients with COVID-19.

- Staff safety should be maintained.

Fig. 1 Ethical challenges and considerations in managing neurologic patients during the COVID-19 pandemic 
present in critically ill children. The pediatric index of mortality and the pediatric risk of mortality have been introduced as references, and they can be used in children [44]. In addition, the natural history of viral infections in children is far different from adults, and treatment failure should not be announced early in the hospital course [44]. In essence, transparent and accessible triage algorithms should be implemented in children, and expected duration of illness and likelihood of return to prior quality of life should be taken into account. In addition, existing or future disabilities should not be a determinant in allocating scarce resources because of their bias in such value-based determinations [44].

\section{Status epilepticus (SE)}

The management of SE patients has changed dramatically due to potential limitation in ventilator and staff capacity. These patients may require ICU admission and should receive equal care with COVID-19 patients. New-onset refractory SE (NORSE) subjects may be more complex cases as they are labor intensive and may need prolonged admissions and immunosuppression. However, decisions should be made individually, and palliative care consultation is necessary [45].

\section{Endovascular treatments (EVT)}

During routine conditions, resource allocation in EVT is performed based on established ethical guidelines, but during COVID-19 pandemic, EVT should be weighed against other uses of scarce medical resources [46]. As a result of the duty to protect community, resources allocated routinely to EVT may be exerted to respond to other essential needs of the community [46]. It should be noted that the COVID-19 is not a contraindication to EVT per se, but it should be avoided for unstable, critically ill patients with COVID-19. In addition, staff safety should be maintained [46]. Ethical challenges and considerations in managing neurologic patients during the COVID-19 pandemic are summarized in (Fig. 1).

\section{NeuroCOVID ward: a new approach}

It has been reported that $40-80 \%$ of COVID-19 patients may present with neurological symptoms [47]. Also, neurodegeneration, neuroinflammation, and demyelination signs are common among COVID-19 patients [48]. As reported by previous studies, a third of COVID-19 patients experience anosmia, hyposmia, and hypogeusia [49]. On the other hand, numerous ICU-requiring neurological conditions, such as stroke and Guillain-Barre syndrome (GBS), have been observed in COVID-19 patients [50,51]. This can create a demand for a neuroCOVID team working in a dedicated ward. As proposed earlier, this team has the potential to gain expertise and supplement clinical care for these patients [52]. Furthermore, neuroCOVID wards/ICUs have the potential to facilitate the management of both neurological patients with COVID-19 and COVID-19 patients with neurological manifestations.

\section{How are physicians and residents being influenced by the COVID-19?}

Protecting healthcare workers (HCWs) during the COVID-19 pandemic is an indispensable part of practice, and personal protective equipment (PPE) is the key way to their safety [53]. Nevertheless, lack of PPE caused fear among HCWs, including neurologist, to go to work as they might be the next victims [54]. But our obligation to provide medical care, even in the face of risks to our own safety [17], made us fight this virus, sometimes unarmed. However, it is still ethically controversial to ask HCWs to counter this threat with insufficient equipment [17]. In terms of education, this pandemic should not hamper the educational process of neurology residents,

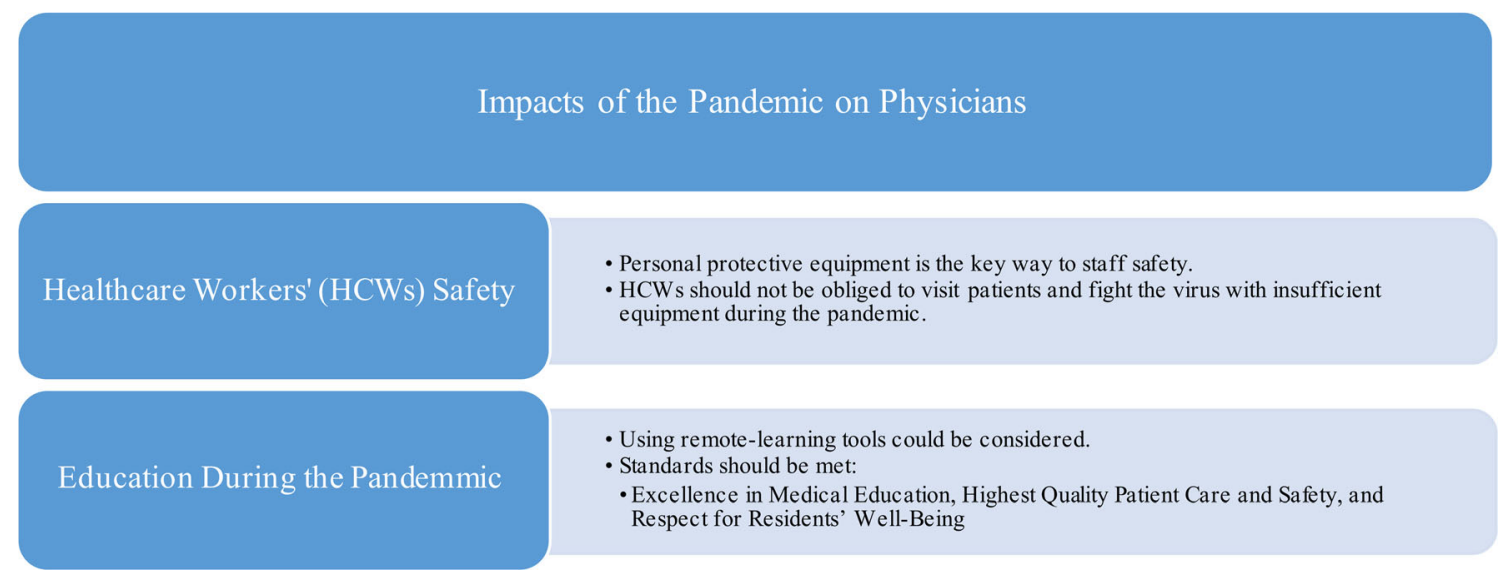

Fig. 2 Impacts of the COVID-19 pandemic on physicians and medical education 
although it may be essential to use remote learning tools [27]. Some trainees may be worried about their personal health and future prospects as a neurologist. Regardless of the situation, these standards should be met: excellence in medical education, highest quality patient care and safety, and respect for residents' well-being [55]. As mentioned in previous studies, many ethical topics can be promoted during this adversity, such as autonomy and distributive justice. Impacts of the COVID-19 pandemic on physicians and medical education are summarized in (Fig. 2).

\section{Second wave of COVID-19}

As it was predicted by previous reports, many countries are now experiencing a second-wave, and they re-entered a second lockdown [56]. This may again give rise to many ethical issues, including the scarcity of ventilators. One possible solution is to transfer patients to other countries/regions with sufficient resources or to flow the resources to critical regions [57]. However, a second-wave planning may be the best option which can even eliminate the need for the abovementioned triage procedures [57]. Also, adequate stockpiles of PPE should be supplied to complement this planning, while health systems ought to develop support mechanism to compensate staff's burnout [58]. Along with this second-wave planning, psychological care needs to be prioritized for both patients and HCWs [57]. As there is flicker of hope with promising COVID-19 vaccines, post-pandemic planning is worth paying attention. The goal of the post-pandemic phase is to address the health and social influence of the pandemic. Also, lessons learned from this pandemic should be identified clearly to prepare health systems for future conditions. In this phase, the psychological impact of the pandemic on people and HCWs needs to be dealt with, resources should be restocked, and the situation should be monitored meticulously [59].

\section{Conclusion}

The COVID-19 pandemic has influenced patients' care, and neurologists have been involved in this daunting situation since the initial days. Although some ethical standards should be balanced to overcome this predicament, principles of ethics ought to be maintained. No doubt are there some challenges, but healthcare workers should endeavor to provide emotional support for the patients, consider non-abandonment of patients, and stick to the main goal of flattening the curve. Although numerous triage protocols have been introduced, each patient should be considered individually. Finally, the management of neurologic patients may be subject to change, as we are facing scarcity of medical resources.
Authors' contribution M.T: Drafting a significant portion of the manuscript, data acquisition (search)

R.A: Drafting a significant portion of the manuscript

A.A: Data acquisition, drafting figures

F.A: Data acquisition, drafting figures

B.S.L: Conception and design of the study, final editing

\section{Compliance with ethical standards}

Conflict of interests The authors declare that they have no conflict of interest.

Ethics approval The manuscript does not contain clinical studies or patient data.

Consent to participate Not applicable

Consent for publication Not applicable

\section{References}

1. Simon V, Ho DD, Karim QA (2006) HIV/AIDS epidemiology, pathogenesis, prevention, and treatment. Lancet 368(9534):489504

2. Schultz CH, Annas GJ (2012) Altering the standard of care in disasters - unnecessary and dangerous. Ann Emerg Med 59(3): 191-19/

3. Berlinger N, Wynia M, Powell T, Hester DM, Milliken A, Fabi R, Jenks N (2020) Ethical framework for health care institutions responding to novel Coronavirus SARS-CoV-2 (COVID-19) guidelines for institutional ethics services responding to COVID19. Safeguarding Communities, Guiding Practice 2

4. Berardelli A, Silani V, Barone P, Calabresi P, Girlanda P, Lopiano L, Massacesi L, Monaco S, Onofrj M, Tassorelli C (2020) Neurology and the COVID-19 emergency. Neurol Sci:1

5. Kim SY, Grady C (2020) Ethics in the time of COVID: what remains the same and what is different. Neurology

6. De Boer ME, Hertogh CM, Dröes R-M, Riphagen II, Jonker C, Eefsting JA (2007) Suffering from dementia-the patient's perspective: a review of the literature. Int Psychogeriatr 19 (6):1021-1039

7. Hensel WF, Wolf LE (2011) Playing god: the legality of plans denying scarce resources to people with disabilities in public health emergencies. Fla L Rev 63:719

8. Richards CT, Crawley LM, Magnus D (2009) Use of neurodevelopmental delay in pediatric solid organ transplant listing decisions: inconsistencies in standards across major pediatric transplant centers. Pediatr Transplant 13 (7):843-850

9. Lee Daugherty-Biddison M, Gwon H, Regenberg A, Schoch-Spana MM, Toner E (2017) Maryland framework for the allocation of scarce life-sustaining medical resources in a catastrophic public health emergency.

10. Emanuel E, Persad G, Upshur R Fair allocation of scarce medical resources in the time of Covid-19 [published online ahead of print March 23, 2020]. N Engl J Med

11. White DB, Katz MH, Luce JM, Lo B (2009) Who should receive life support during a public health emergency? Using ethical principles to improve allocation decisions. Ann Intern Med 150 (2): $132-138$

12. Persad G, Wertheimer A, Emanuel EJ (2009) Principles for allocation of scarce medical interventions. Lancet 373 (9661):423-431 
13. Shuman AG (2020) Navigating the Ethics of COVID-19 in otolaryngology. Otolaryngology-Head and Neck Surgery: 0194599820920850

14. Shuman AG, Pentz RD (2020) Cancer research ethics and COVID19. The Oncologist

15. Chan KY, Chan ML (2018) Enhanced psychosocial support as important component of neuro-palliative service. Ann Palliat Med 7 (3):355-358. doi:10.21037/apm.2017.08.19

16. Marchiano EJ, Birkeland AC, Swiecicki PL, Spector-Bagdady K, Shuman AG (2018) Revisiting expectations in an era of precision oncology. The oncologist 23 (3):386

17. Nelson SE (2020) COVID-19 and ethics in the ICU. Springer,

18. Wakam GK, Montgomery JR, Biesterveld BE, Brown CS (2020) Not dying alone - modern compassionate care in the Covid-19 pandemic. N Engl J Med 382 (24):e88

19. Lamas D (2020) Opinion| I'm on the front lines. I have no plan for this. The New York Times

20. Hammers DB, Stolywk R, Harder L, Cullum CM (2020) A survey of international clinical teleneuropsychology service provision prior to and in the context of COVID-19. Clin Neuropsychol:1-17

21. Brizzi K, Creutzfeldt CJ (2018) Neuropalliative care: a practical guide for the neurologist. Semin Neurol 38 (5):569-575. doi: 10.1055/s-0038-1668074

22. Swain A, Bhagat H, Sahni N, Salunke P (2016) Mechanical ventilation in neurological and neurosurgical patients. Neurol India 64 (3): 485

23. Biddison ELD, Faden R, Gwon HS, Mareiniss DP, Regenberg AC, Schoch-Spana M, Schwartz J, Toner ES (2019) Too many patients ... a framework to guide statewide allocation of scarce mechanical ventilation during disasters. Chest 155 (4):848-854

24. Emanuel EJ, Persad G, Upshur R, Thome B, Parker M, Glickman A, Zhang C, Boyle C, Smith M, Phillips JP (2020) Fair allocation of scarce medical resources in the time of Covid-19. Mass Medical Soc,

25. Association BM (2020) COVID-19-ethical issues. A guidance note 1

26. Yang X, Yu Y, Xu J, Shu H, Liu H, Wu Y, Zhang L, Yu Z, Fang M, Yu T (2020) Clinical course and outcomes of critically ill patients with SARS-CoV-2 pneumonia in Wuhan, China: a single-centered, retrospective, observational study. Lancet Respir Med

27. Rubin MA, Bonnie RJ, Epstein L, Hemphill C, Kirschen M, Lewis A, Suarez JI, Committee NCSE (2020) AAN position statement: the COVID-19 pandemic and the ethical duties of the neurologist. Neurology

28. Rubin MA, Truog RD (2017) What to do when there aren't enough beds in the PICU. AMA J Ethics 19 (2):157-163

29. Peterson A, Largent EA, Karlawish J (2020) Ethics of reallocating ventilators in the covid-19 pandemic. Bmj 369

30. Truog RD, Mitchell C, Daley GQ (2020) The toughest triageallocating ventilators in a pandemic. N Engl J Med 382 (21): 1973-1975

31. White DB, Lo B (2020) A framework for rationing ventilators and critical care beds during the COVID-19 pandemic. Jama 323 (18): 1773-1774

32. Stall N, Sinha S. COVID-19 isn't the only thing that's gone viral. Ageism has, too. The globe and the mirror. Available at: https:// www.the-globeandmail.com/opinion/article-covid-19-isnt-the-only-thing-thats-gone-viral-age-ism-has-too/.

33. Smith EE, Couillard P, Fisk JD, Ismail Z, Montero-Odasso M, Robillard JM, Vedel I, Sivananthan S, Gauthier S (2020) Pandemic dementia scarce resource allocation. Can Geriatr J 23 (3): 216

34. Geddes MR, O'Connell ME, Fisk JD, Gauthier S, Camicioli R, Ismail Z, COVID-19 ASoCTFoDCBPf (2020) Remote cognitive and behavioral assessment: report of the Alzheimer Society of Canada Task Force on dementia care best practices for COVID-
19. Alzheimer's \& Dementia: Diagnosis, Assessment \& Disease Monitoring 12 (1):e12111

35. Gupta R, Kumar VM, Tripathi M, Datta K, Narayana M, Sarmah KR, Bhatia M, Devnani P, Das S, Shrivastava D (2020) Guidelines of the Indian Society for Sleep Research (ISSR) for practice of sleep medicine during COVID-19. Sleep and Vigilance:1-12

36. Wijnands JMA, Zhu F, Kingwell E, Fisk JD, Evans C, Marrie RA, Zhao Y, Tremlett H (2018) Disease-modifying drugs for multiple sclerosis and infection risk: a cohort study. J Neurol Neurosurg Psychiatry 89 (10):1050-1056. https://doi.org/10.1136/jnnp-2017317493

37. Shahidi-Dadras M, Abdollahimajd F, Ohadi L, Tabary M, Araghi F, Mozafari N, Toosi P, Dadkhahfar S (2020) COVID-19 in pemphigus vulgaris patients with previous rituximab therapy: a telemedicine experience. J Dermatol Treat:1-2

38. Sahraian MA, Gheini MR, Rezaeimanesh N, Ghajarzadeh M, Naser Moghadasi A (2020) Knowledge regarding COVID-19 pandemic in patients with multiple sclerosis (MS): a report from Iran. Mult Scler Relat Disord 42:102193. https://doi.org/10.1016/j.msard. 2020.102193

39. Seyed Ahadi M, Sahraian MA, Rezaeimanesh N, Moghadasi AN Psychiatric advice during COVID-19 pandemic for patients with multiple sclerosis. Iran J Psychiatry Behav Sci 14 (2)

40. Markus HS, Brainin M (2020) COVID-19 and stroke-A global World Stroke Organization perspective. Int J Stroke 15 (4):361-364

41. Aguiar de Sousa D, Sandset EC, Elkind MS (2020) The curious case of the missing strokes during the COVID-19 pandemic. Am Heart Assoc,

42. Leira EC, Russman AN, Biller J, Brown DL, Bushnell CD, Caso V, Chamorro A, Creutzfeldt CJ, Cruz-Flores S, Elkind MS (2020) Preserving stroke care during the COVID-19 pandemic: potential issues and solutions. Neurology

43. Curtis JR, Kross EK, Stapleton RD (2020) The importance of addressing advance care planning and decisions about do-notresuscitate orders during novel coronavirus 2019 (COVID-19). Jama 323 (18): 1771-1772

44. Laventhal NT, Graham RJ, Rasmussen SA, Urion DK, Kang PB (2020) Ethical decision-making for children with neuromuscular disorders in the COVID-19 crisis. Neurology

45. Kinney MO, Brigo F, Kaplan PW (2020) Optimizing status epilepticus care during the COVID-19 pandemic. Epilepsy Behav 109: 107124. https://doi.org/10.1016/j.yebeh.2020.107124

46. Smith MS, Bonomo J, Knight WA IV, Prestigiacomo CJ, Richards CT, Ramser E, Adeoye O, Bertsch S, Shirani P, Vagal A (2020) Endovascular therapy for patients with acute ischemic stroke during the COVID-19 pandemic: a proposed algorithm. Stroke 51(6): 1902-1909

47. Mao L, Jin H, Wang M, Hu Y, Chen S, He Q, Chang J, Hong C, Zhou Y, Wang D, Miao X, Li Y, Hu B (2020) Neurologic manifestations of hospitalized patients with coronavirus disease 2019 in Wuhan, China. JAMA Neurol 77 (6):683-690. https://doi.org/10. 1001/jamaneurol.2020.1127

48. Zanin L, Saraceno G, Panciani PP, Renisi G, Signorini L, Migliorati $\mathrm{K}$, Fontanella MM (2020) SARS-CoV-2 can induce brain and spine demyelinating lesions. Acta Neurochir (Wien) 162 (7):1491-1494. https://doi.org/10.1007/s00701-020-04374-x

49. Nouchi A, Chastang J, Miyara M, Lejeune J, Soares A, Ibanez G, Saadoun D, Morélot-Panzini C, Similowski T, Amoura Z (2020) Prevalence of hyposmia and hypogeusia in 390 COVID-19 hospitalized patients and outpatients: a cross-sectional study. Eur J Clin Microbiol Infect Dis:1-7

50. Toscano G, Palmerini F, Ravaglia S, Ruiz L, Invernizzi P, Cuzzoni MG, Franciotta D, Baldanti F, Daturi R, Postorino P (2020) GuillainBarré syndrome associated with SARS-CoV-2. N Engl J Med 
51. Avula A, Nalleballe K, Narula N, Sapozhnikov S, Dandu V, Toom S, Glaser A, Elsayegh D (2020) COVID-19 presenting as stroke. Brain, behavior, and immunity

52. McAlpine LS, Zubair AS, Moeller J, Baehring J, Spudich S (2020) Lessons from a neurology consult service for patients with COVID19. The Lancet Neurology 19 (10):806-807

53. Gheisari M, Araghi F, Moravvej H, Tabary M, Dadkhahfar S (2020) Skin reactions to non-glove personal protective equipment: an Emerging Issue in the COVID-19 Pandemic. J Eur Acad Dermatol Venereol

54. Turale S, Meechamnan C, Kunaviktikul W (2020) Challenging times: ethics, nursing and the COVID-19 pandemic. Int Nurs Rev 67 (2):164-167

55. Compact between resident physicians and their teachers. Available at: www.aamc.org/residentcompact. Accessed October 8, 2020.
56. Looi M-K (2020) Covid-19: is a second wave hitting Europe? bmj 371

57. Wirth M, Rauschenbach L, Hurwitz B, Schmiedebach H-P, Herdt JA (2020) The meaning of care and ethics to mitigate the harshness of triage in second-wave scenario planning during the COVID-19 pandemic. Am J Bioeth 20 (7):W17-W19

58. Middleton J, Lopes H, Michelson K, Reid J (2020) Planning for a second wave pandemic of COVID-19 and planning for winter. Int J Public Health:1-3

59. Organization WH (2009) Pandemic influenza preparedness and response: a WHO guidance document. World Health Organization

Publisher's note Springer Nature remains neutral with regard to jurisdictional claims in published maps and institutional affiliations. 